\title{
Strong teeth make strong kids
}

Oral-B is on a mission to support UK parents and carers to adopt appropriate home-based oral health behaviours and thereby reduce the number of children with toothache and dental problems - all through its \#StrongTeethMakeStrongKids campaign. The oral health experts from Oral-B and the University of Leeds, have launched a research and education programme to give dental professionals and parents the right support to prevent these dental health issues from now on. Peter Day, Associate Professor and Consultant in Paediatric Dentistry at the University of Leeds, says about the research: 'Our research explored how dental teams can best support parents of young children to adopt appropriate oral health behaviours at home. We have examined the literature and undertaken qualitative interviews and focus groups to identify the challenges parents and dental teams face. These findings have provided the blueprint for Strong Teeth oral health intervention.'

'We are working closely with the UK's dental professionals by aiming to provide up

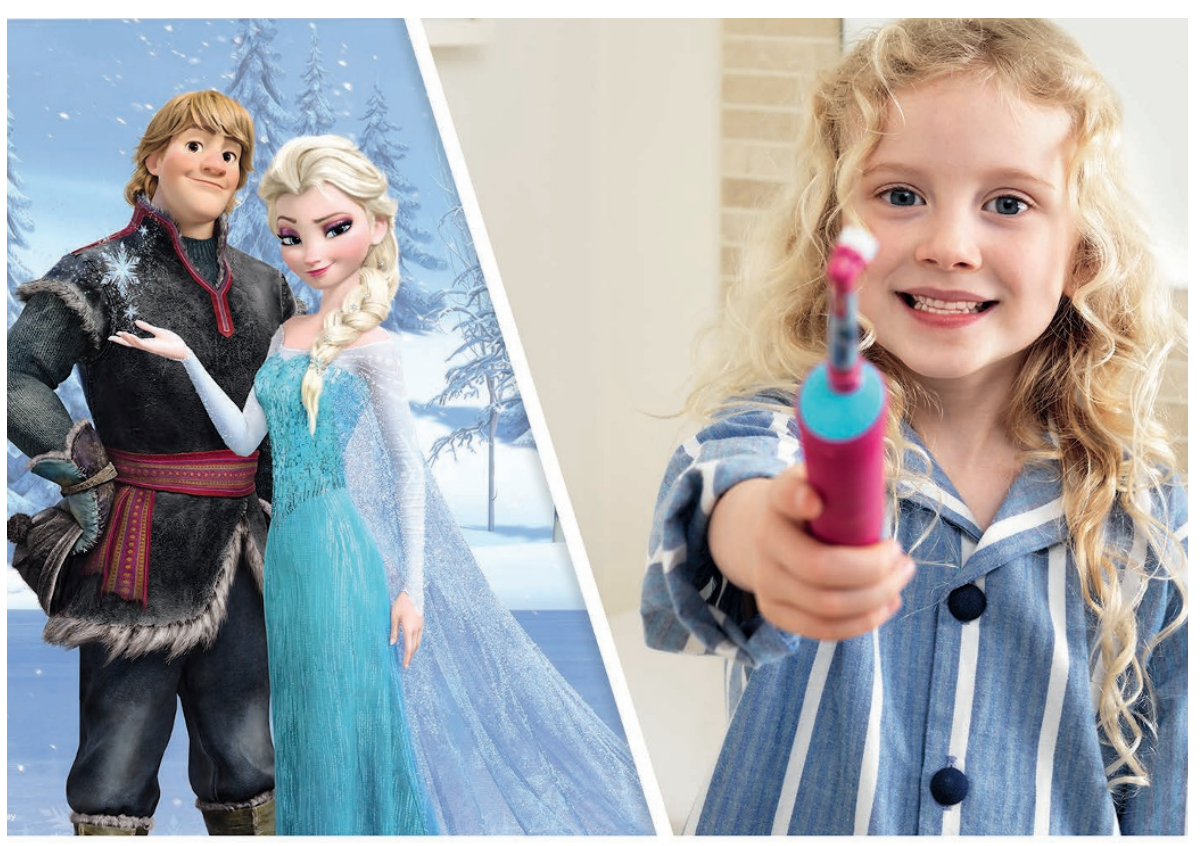

to 20,000 dental professionals this year with simple and engaging educational materials for parents and their children during routine check-ups. Oral-B is committed to take on this challenge to sustainably improve the situation in the UK', says Jane Kidson, Oral-B Professional Team Leader UK and Ireland.

Combined with the right dental care products, these positive oral health messages are designed to encourage parents to lead the way, so they can see that these oral health issues are mostly preventable with simple changes to their families' daily oral care routine.

Here is a snapshot of the brand's educational materials that dental professionals can use to support the conversations that they are having with parents and carers:

1. Brushing from first tooth to five years

2. Friends and family can support healthy habits

3. Make brushing fun for children

4. Healthy eating can help protect teeth.

Maintaining good oral health and establishing the right healthy habits early on will help children progress along the key 'Strong Teeth' milestones. They include their first dental visit, the arrival of their first tooth or teeth, and then the first time they use an electric power toothbrush (from the age of three onwards).

\section{Feeling protective}

The British Dental Conference and Dentistry Show provided the backdrop for the latest product launch by Philips Oral Healthcare. Its latest ProtectiveClean toothbrush range features Sonicare's unique sonic cleaning technology at its core; so like its predecessors it produces 32,000 brush sweeps a minute, creating the dynamic fluid activity - aerating saliva and toothpaste - for a penetrative clean, for which it is famed.

The new ProtectiveClean range also features a two-minute timer and a pressure sensor, which provides real time feedback to ensure that patients are not brushing too hard, thereby minimising their risk of gum damage and gingival recession. Thanks to its clinically efficacious Optimal Plaque Control brush head ProtectiveClean is also clinically proven to remove seven times more plaque than a manual toothbrush. The brush-head also features new BrushSync radio frequency identification device (RFID) technology to monitor usage and alert the patient when it is time to replace it with a reviving new one and ramp up the efficacy of the brush to its full capacity again.

A strong differentiating factor of the new brush, which should appeal to dental professionals is that the ProtectiveClean range addresses multiple price points, making good oral hygiene more accessible to a far wider cohort of patients. The expected retail sales price of the entry-level handle, ProtectiveClean 4300, is £79.99.

The brush includes one inbuilt cleaning mode, with two intensity settings - high and low - to provide users with a tailored clean. The handle comes with an Optimal Plaque Defence brush head, charger, travel case, features BrushSync technology and is available in range of colours, light pink, light blue and black.

Philips Sonicare ProtectiveClean 6100 features inbuilt pressures sensors, includes three cleaning modes; clean, gum care and white, and three intensity settings; high, low and medium. The brush also includes two BrushSync features, a brush head replacement reminder and brush head mode pairing, to provide users with a tailored clean. The handle comes with two Optimal White brush heads, a travel case and charger.

For more information, please visit http://www.philips.co.uk/sonicare. 